\title{
Usefulness of Semi-cylindrical Beam Spoiler in Treatment of Early Glottic Cancer Using 6 MV Photon Beam
}

\author{
SEONG SOO SHIN ${ }^{1 *}$, SOHYUN AHN ${ }^{2 *}$, SEUNG-HYUP BAEK ${ }^{3}$, \\ JUNGWON KWAK ${ }^{4}$, JIN SUNG KIM ${ }^{2}$ and WOO SANG AHN ${ }^{1}$ \\ ${ }^{1}$ Department of Radiation Oncology, Gangneung Asan Hospital, \\ University of Ulsan College of Medicine, Gangneung, Republic of Korea; \\ ${ }^{2}$ Department of Radiation Oncology, Yonsei University College of Medicine, Seoul, Republic of Korea; \\ ${ }^{3}$ Department of Integrative Medicine, Yonsei University, Seoul, Republic of Korea; \\ ${ }^{4}$ Department of Radiation Oncology, Asan Medical Center, \\ University of Ulsan College of Medicine, Seoul, Republic of Korea
}

\begin{abstract}
Background/Aim: To assess the dosimetric influence of a semi-cylindrical beam spoiler ( $s C B S)$ for the treatment of early glottic cancer using a $6 \mathrm{MV}$ photon beam. Patients and Methods: The $2 \mathrm{D}$ dose distributions were also calculated and measured with and without the sCBS and with a $0.5 \mathrm{~cm}$ thick bolus. A retrospective study of 8 patients treated between 2012 and 2018 was performed. Results: The $2 D$ dose distributions obtained from the treatment planning system (TPS) and film measurements were in good agreement. In the planning study, the $V_{95 \%}, V_{100 \%}$, conformal index (CI), and homogeneity index (HI) of all pPTVs for the sCBS plans were better than those for the open field plans $(p<0.01)$. Especially, $s C B S$ plans had better skin sparing effect than bolus plans $(p<0.05)$. Conclusion: The sCBS of the $6 \mathrm{MV}$ photon beam could be a useful tool for the treatment of early glottic cancers.
\end{abstract}

The early glottic squamous cell carcinoma has a favorable prognosis. The treatment modes for early glottic cancer include surgery and radiotherapy (1-6). Radiotherapy has been traditionally performed with cobalt-60 or low-energy megavoltage (2-4 MV) photon beams, as noted in published data $(5,7-10)$. The five-year survival rates range from 80 -

This article is freely accessible online.

*These Authors contributed equally to this study.

Correspondence to: Woo Sang Ahn, Department of Radiation Oncology, Gangneung Asan Hospital, University of Ulsan College of Medicine, Gangneung 25440, Republic of Korea. Tel: +82 336105315, e-mail: anidol@ulsan.ac.kr

Key Words: Beam spoiler, glottic cancer, photon beam, $6 \mathrm{MV}$, radiotherapy.
$85 \%$, and local control rates of approximately $95 \%, 85 \%$, and $75 \%$ have been achieved for stages $\mathrm{T} 1, \mathrm{~T} 2 \mathrm{a}$, and $\mathrm{T} 2 \mathrm{~b}$, respectively.

The conventional radiation therapy of stage $\mathrm{T} 1$ lesions mainly include the parallel-opposed or slightly oblique lateral field technique using 2-4 MV photon beams. As radiotherapy equipment with advanced performances have been developed, low-energy MV X-ray machines have been decommissioned or minimally utilized in many radiation oncology departments. Currently, $6 \mathrm{MV}$ or higher-energy Xrays are mostly administered for the treatment of early glottic cancer (11-13).

The use of high-energy photon beams of $6 \mathrm{MV}$ or more has the advantage of the skin sparing effect while delivering sufficient doses to tumors in the deep regions owing to the high penetration power of the beams. However, as skin tumors or glottic cancers have a target volume up to the site adjacent to the skin, there is a possibility that insufficient doses may be delivered to the tumor sites because of the skin sparing effects of the high-energy photon beams. This is because the electron equilibrium is not formed in tissues that are in contact with air. Therefore, there is a possibility that the doses may not be sufficiently delivered to the mucosal or anterior commissure region in contact with the laryngeal air cavity region (14).

To solve the problem of insufficient dose delivery in the superficial regions, a bolus, i.e., a tissue-equivalent material, is used as the simplest method in clinical practice (15). The bolus is mainly used to compensate for the insufficient electronic balance in areas where the tissue is in contact with air. The use of a bolus can deliver a sufficient dose to a target volume adjacent to the surface, but there is a disadvantage that the skin sparing effect may disappear and serious skin reactions might occur. Therefore, when a bolus is used in clinical practice, it is sometimes applied at 


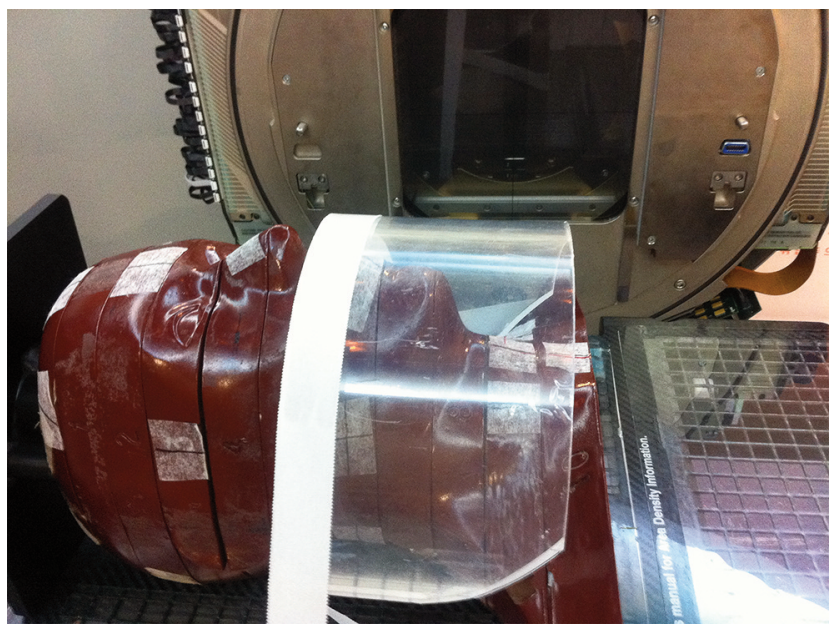

Figure 1. Semi-cylindrical beam spoiler developed with the lateral parallel-opposed fields of a $6 \mathrm{MV}$ photon beam for early glottic cancer treatment.

intervals of 2 or 3 days to minimize skin reactions. Another problem with this approach is that in the case of severe surface curvatures, such as in head and neck cancers, it is difficult to ensure complete adherence of the bolus to the skin surface, and the air-gap effect may result in a dose distribution different from expectation.

Another option that can be considered for dose escalation in superficial regions is the beam spoiler. Originally, the beam spoiler was designed to increase the skin dose in skin cancers or invasive tumors from head and neck cancer to the superficial layers. Currently, the beam spoiler is mainly used for total body irradiation (TBI) and total skin electron beam therapy (TSEBT), where it is placed between the patient and the linear accelerator such that secondary electrons generated by the beam spoiler enter the patient's skin. The concept of a planar-type beam spoiler has been reported in several works. This design was investigated to ensure adequate dosage to the superficial tissues of the head and neck when using high-energy photon beams. By measuring the percent depth dose (PDD) for a $6 \mathrm{MV}$ photon beam after placing a $1.2 \mathrm{~cm}$ thick planar-type beam spoiler at a distance of $7 \mathrm{~cm}$ from the water surface, Lee et al. showed that the dose at a $5 \mathrm{~mm}$ depth was more than $90 \%$ of the maximum dose (16).

The surface dose generally increases as the field size increases when using a beam spoiler. Kassaee $e t$ al. showed that the planar-type spoiler increases the dose within the build-up region as the distance between the spoiler and water surface decreases and field size increases (17). However, a sufficient dose enhancement within the build-up region was particularly ineffective for small field size such as $5 \times 5 \mathrm{~cm}^{2}$.

In radiotherapy for early glottic cancer, field sizes of $5 \times 5$ or $6 \times 6 \mathrm{~cm}^{2}$ are typically used. For sufficient dose enhancement in the build-up region for such relatively small field sizes, the beam spoiler should be placed as close to the surface as possible. However, the planar-type beam spoiler is limited by the fact that it is difficult to position the device close to the skin surface owing to the presence of the patient's shoulder.

In this study, a dedicated semi-cylindrical beam spoiler (sCBS) that satisfied the following clinical requirements was developed for the radiotherapy of early glottic cancer: First, it should provide a sufficient dose to the target volume for early glottic cancer, especially the type involving the anterior commissure; the minimum required dose at $5 \mathrm{~mm}$ should be at least $90 \%$ for field sizes of $5 \times 5$ to $6 \times 6 \mathrm{~cm}^{2}$. Second, the device should allow for the skin sparing effect. Third, the spoiler should be easy to set up. The dosimetric effects of the sCBS using a $6 \mathrm{MV}$ photon beam were thus evaluated in this work.

\section{Patients and Methods}

Semi-cylindrical beam spoiler. We developed the sCBS shown in Figure 1 for the radiation treatment of early glottic cancer using the parallel-opposed lateral field of a $6 \mathrm{MV}$ photon beam. The sCBS consists of a semi-cylindrical substrate of polymethylmethacrylate (PMMA), with $18 \mathrm{~cm}$ inner diameter and $0.5 \mathrm{~cm}$ thickness.

Percent depth dose measurements. To verify the effect of dose build-up enhanced by the sCBS, the PDDs in water phantom were measured using a plane-parallel ionization chamber (PPC40, IBA Dosimetry, Schwarzenbruck, Germany), as shown in Figure 2. Measurements of the relative doses in the build-up region at the beam spoiler-to-source distance of 2, 5, 7, and $10 \mathrm{~cm}$ were taken at fixed $100 \mathrm{~cm}$ source-to-surface distance (SSD), with the $6 \mathrm{MV}$ photon beam from the Varian Truebeam linear accelerator. All measurements were performed by using a field size of $5 \times 5 \mathrm{~cm}^{2}$, and are mainly used for the radiotherapy of early glottic cancer.

\section{Phantom study.}

Contour and planning. A computed tomography (CT) scanner was used to acquire images of the Rando phantom (The Phantom Laboratory, Salem, NY, USA), which comprises a skeleton and plastic tissue-equivalent materials. The CT images were obtained in the axial mode, with a slice thickness of $2.5 \mathrm{~mm}$, and transferred to a radiation treatment planning system (Eclipse version 8.9, Varian Medical Systems, CA, USA). A radiation oncologist then contoured the clinical target volume (CTV) of the glottic cancer and added the appropriate margins to the CTV for generating the planning target volume (PTV). An isocenter was set at the center in the PTV for radiotherapy. Three treatment plans were planned with the parallelopposed lateral fields using the $6 \mathrm{MV}$ photon beam: 1) without the cylindrical beam spoiler (open beam), 2) with the cylindrical beam spoiler, and 3) with a bolus of $0.5 \mathrm{~cm}$ thickness. The field size of $5 \times 5 \mathrm{~cm}^{2}$ was chosen for all plans, and optimized wedges were employed to ensure homogeneous dose distributions in the glottic area. The 3D pencil beam superposition-convolution algorithm (analytical anisotropic algorithm: AAA) was used to perform the dose calculations with a grid size of $2.5 \mathrm{~mm}$. 


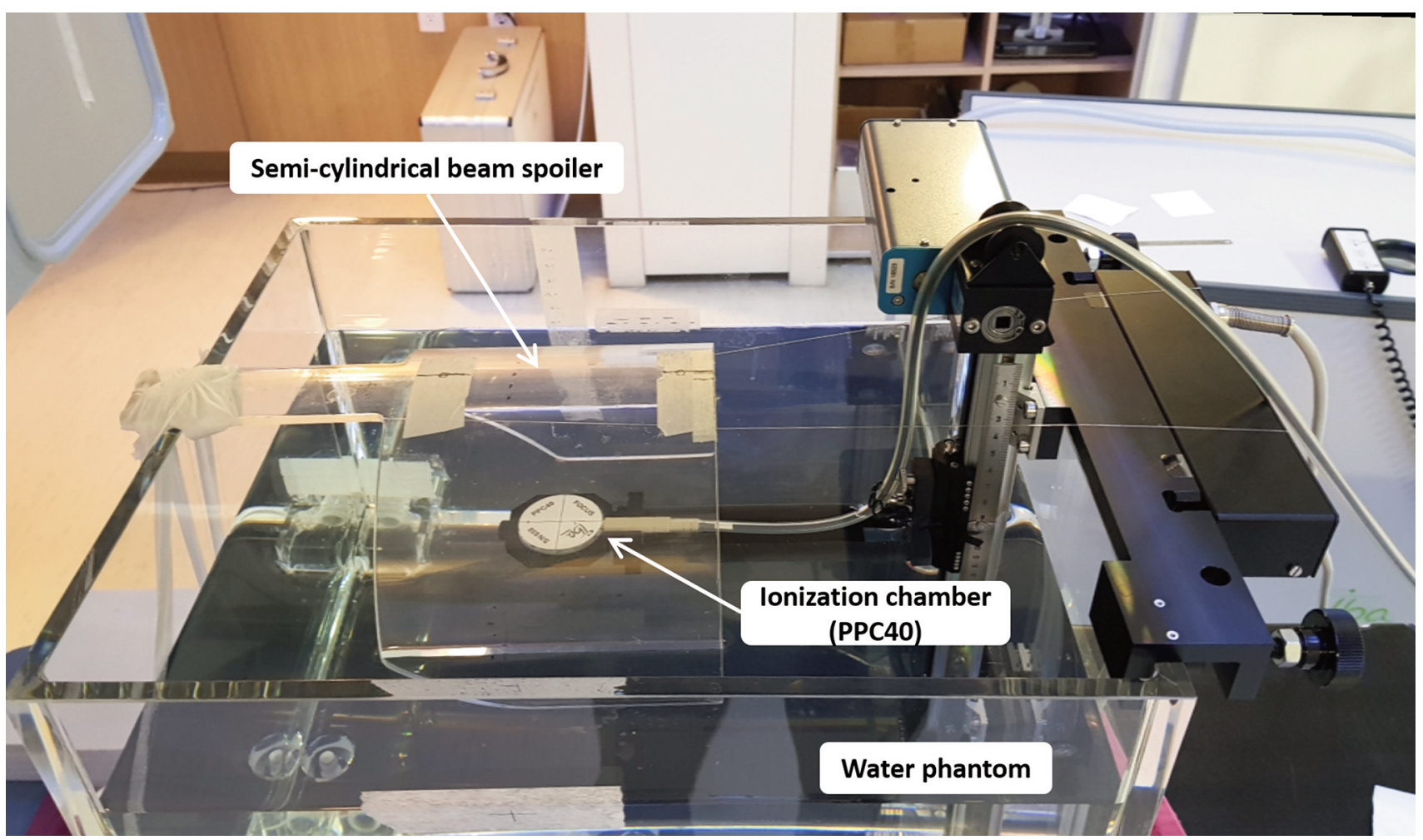

Figure 2. Experimental setup for measuring the percent depth dose in the build-up region using a PPC40 plane-parallel ionization chamber.

$2 D$ dose distributions. To verify the dose distributions calculated from the treatment planning system (TPS), the data were compared with those from Gafchromic EBT2 films measurements used with the Rando phantom. The lateral profiles were measured for the field size of $5 \times 5 \mathrm{~cm}^{2}$ under the three conditions noted above. The rootmean-squared error (RMSE) was used to analyze the differences between the measured and calculated lateral beam profiles along the central axis. To evaluate the quantitatively measured and calculated dose distributions, a gamma index analysis was performed using a commercial software package (OmniPro I'mRT, IBA Dosimetry, Schwarzenbruck, Germany). The gamma index with the individual acceptance criteria of $3 \%$ of the dose difference and $3 \mathrm{~mm}$ distance to agreement (DTA) were used for the evaluations.

Treatment planning study. Eight patients with early glottic cancer were approved by the Gangneung Asan Hospital institutional review board (IRB, No. GNAH 2021-01-003). The informed consent was waived by the IRB protocol. Only image and dose-volume data were used in this study. All patient-related health information was removed for the study.

The CT images of eight patients with early glottic cancer involving the anterior commissure were imported into the TPS. On each slice of the CT scan, the target volume and organ at risk were defined by a radiation oncologist. The patients were to be treated with the parallel-opposed lateral fields of the $6 \mathrm{MV}$ photon beam. Because the gross tumor volume (GTV) is close to the skin, the general dose-volume histograms (DVHs) are unclear for the effects of the spoiler. In this study, to evaluate the dose distributions at low depths for the sCBS, the open beam (OB), bolus plans, partial planning target volumes (pPTVs), and partial skin regions (SkinpPTVs) were defined in $2 \mathrm{~mm}$ depth increments. The conformal index (CI), homogeneity index (HI), and maximum dose volume $\mathrm{V}_{105 \%}$ for the pPTVs as well as the mean dose $\left(\mathrm{D}_{\text {mean }}\right)$ for the partial skin regions were selected as the dosimetric parameters. The dose distributions of the photon beam for all parallel-opposed lateral field plans were calculated using the AAA (version 8.9, Varian Medical Systems, Palo Alto, CA, USA) with a grid size of $2 \mathrm{~mm}$. All plans were then normalized to cover at least $95 \%$ of the PTV.

Statistical analysis. The dosimetric differences were analyzed among the three treatment techniques for the eight patients using a one-way ANOVA test. The differences were considered at the 0.05 level of significance ( $p$-value). All statistical analyses were performed using the SPSS software, version 15.0 (SPSS Inc., Chicago, IL, USA).

\section{Results}

Measured relative doses in the build-up region. Figure 3 shows the measured central axis depth doses in the water phantom as a function of the beam spoiler-to-surface distances for a field size of $5 \times 5 \mathrm{~cm}^{2}$ with the $6 \mathrm{MV}$ photon beam. All the measured data were normalized at the depth of $3 \mathrm{~cm}$, where sufficient 


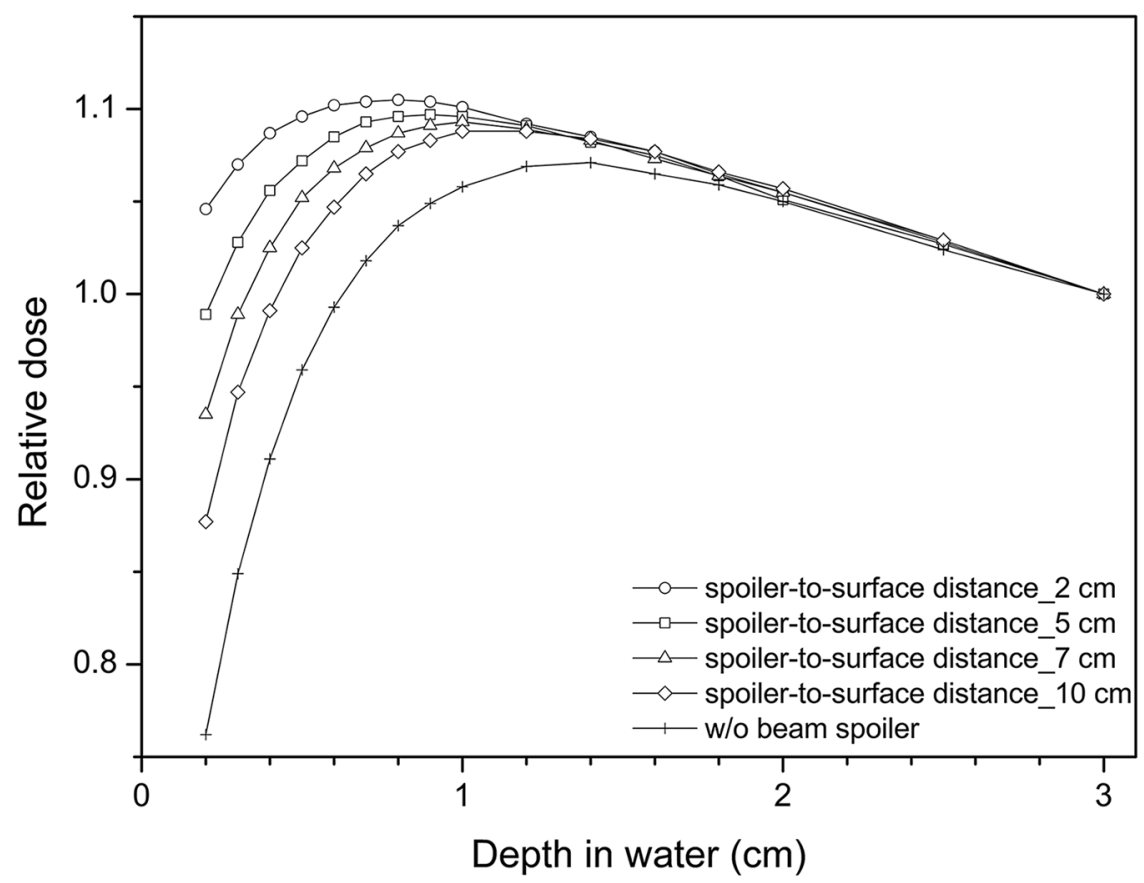

Figure 3. Measured percent depth doses as functions of the spoiler-to-surface distances for a field size of $5 \times 5 \mathrm{~cm}^{2}$ of the $6 \mathrm{MV}$ photon beam.

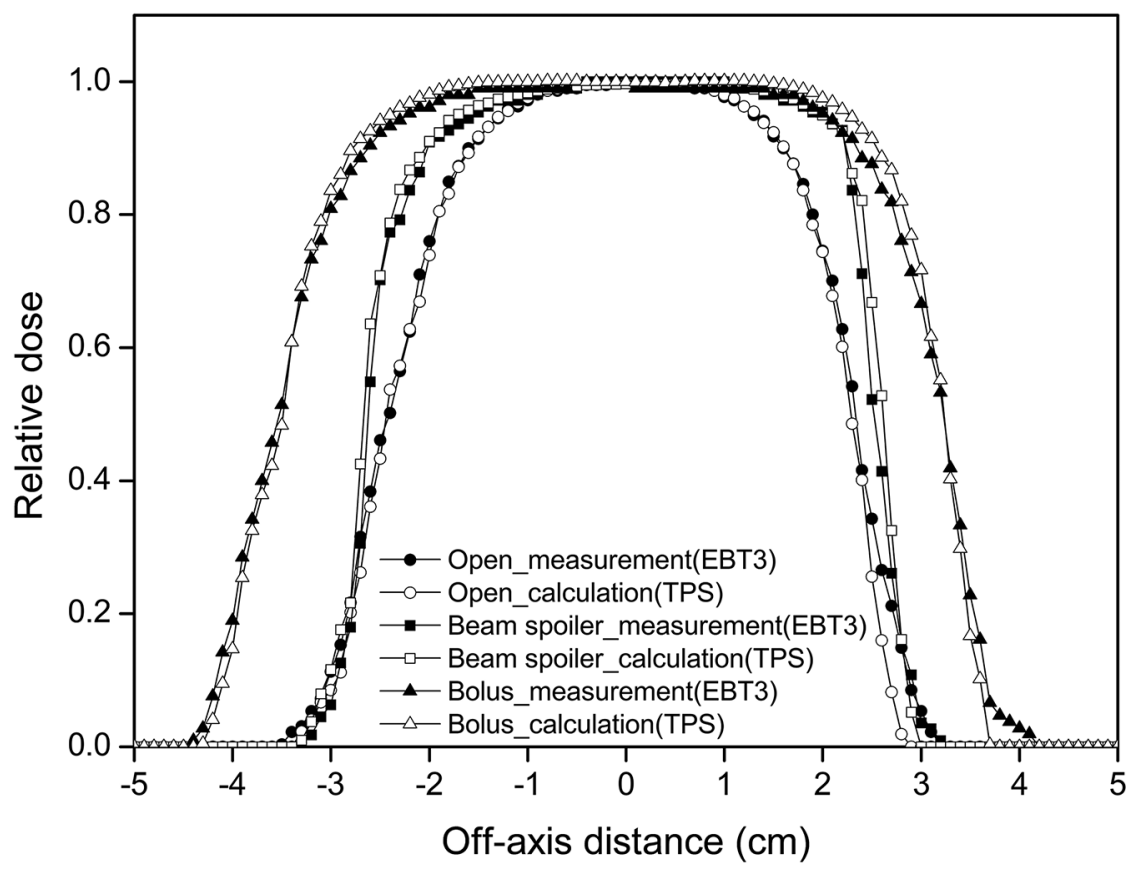

Figure 4. Lateral dose profiles for $5 \times 5 \mathrm{~cm}^{2}$ lateral parallel-opposed fields of the $6 \mathrm{MV}$ photon beam with and without beam spoiler and with an open beam.

electronic equilibrium was achieved. The use of the sCBS shows the effects of dose enhancement within the build-up region, while there are no significant influences on the depth doses beyond the maximum depth dose for the OB case. The relative doses for the spoiler-to-surface distances of 2,5,7, and $10 \mathrm{~cm}$ at a depth of $0.5 \mathrm{~cm}$ were $1.094,1.072,1.052$, and 1.025 , respectively. This is higher than the relative dose at the reference depth of $3 \mathrm{~cm}$, which is the point of normalization. 

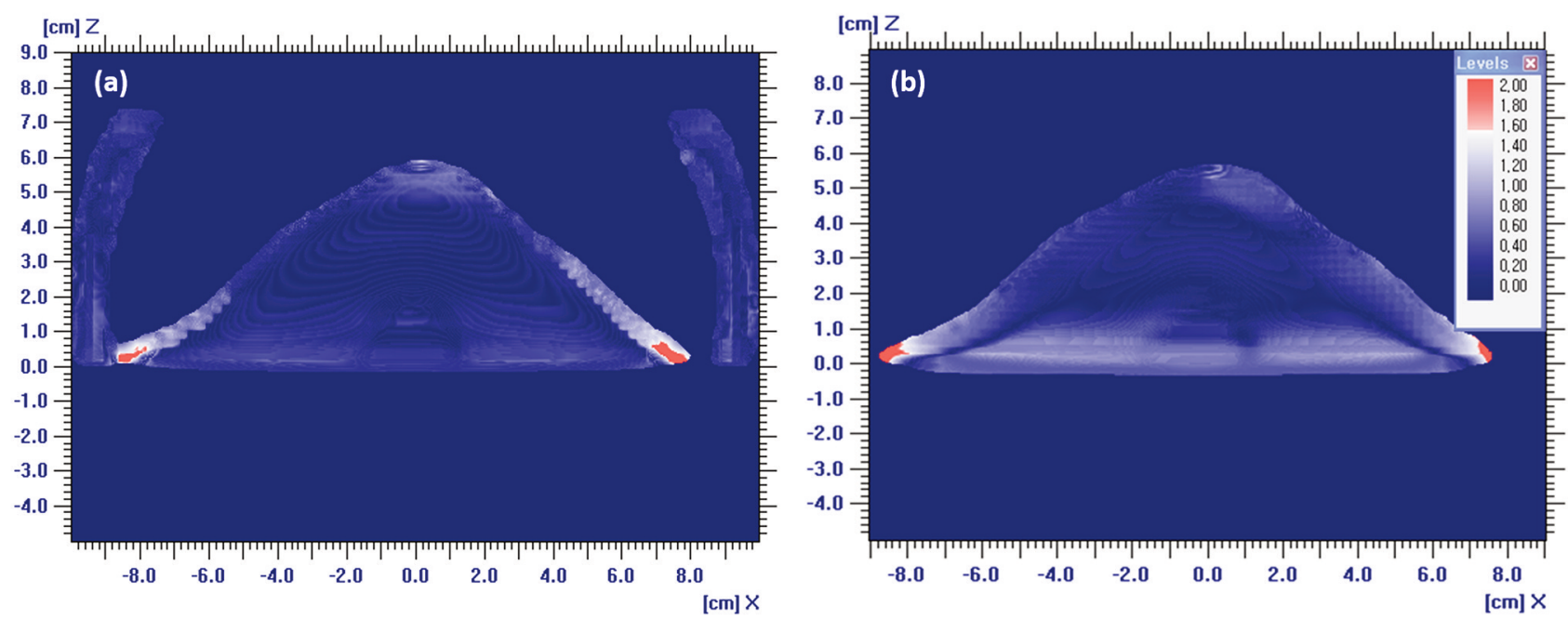

Figure 5. Gamma index evaluation for measured and calculated $2 D$ dose distributions of the $6 M V$ photon beam (A) with and (B) without the semicylindrical beam spoiler.

Table I. Summary of all investigated dose-volume histogram (DVH) parameters as mean values \pm standard deviations.

\begin{tabular}{|c|c|c|c|c|c|c|c|}
\hline Plan & Parameters & pPTV & $\mathrm{pPTV}_{0-2 \mathrm{~mm}}$ & $\mathrm{pPTV}_{2-4 \mathrm{~mm}}$ & 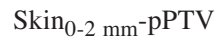 & Skin $_{2-4 ~ m m}-$ pPTV & Skin $_{4-6 ~ m m}$-pPTV \\
\hline \multirow[t]{6}{*}{ sCBS } & $\mathrm{V}_{95 \%}(\%)$ & $97.87 \pm 1.92$ & $93.09 \pm 7.21$ & $99.95 \pm 0.09$ & & & \\
\hline & $\mathrm{V}_{100 \%}(\%)$ & $82.63 \pm 9.40$ & $58.34 \pm 26.17$ & $91.60 \pm 8.90$ & & & \\
\hline & $\mathrm{V}_{105 \%}(\%)$ & $24.97 \pm 17.91$ & $13.96 \pm 26.54$ & $26.70 \pm 27.63$ & & & \\
\hline & CI & $0.98 \pm 0.02$ & $0.93 \pm 0.07$ & $1.00 \pm 0.00$ & & & \\
\hline & $\mathrm{HI}$ & $1.10 \pm 0.01$ & $1.09 \pm 0.02$ & $1.06 \pm 0.01$ & & & \\
\hline & $\mathrm{D}_{\text {mean }}(\%)$ & & & & $85.13 \pm 2.06$ & $93.38 \pm 1.75$ & $100.71 \pm 1.60$ \\
\hline \multirow[t]{6}{*}{ OB } & $\mathrm{V}_{95 \%}(\%)$ & $79.97 \pm 12.64$ & $40.29 \pm 31.35$ & $69.54 \pm 30.36$ & & & \\
\hline & $\mathrm{V}_{100 \%}(\%)$ & $61.42 \pm 17.78$ & $26.01 \pm 32.68$ & $48.61 \pm 36.18$ & & & \\
\hline & $\mathrm{V}_{105 \%}(\%)$ & $11.27 \pm 13.31$ & $5.98 \pm 16.26$ & $12.50 \pm 22.29$ & & & \\
\hline & CI & $0.80 \pm 0.13$ & $0.40 \pm 0.31$ & $0.70 \pm 0.30$ & & & \\
\hline & $\mathrm{HI}$ & $1.37 \pm 0.17$ & $1.46 \pm 0.18$ & $1.16 \pm 0.07$ & & & \\
\hline & $\mathrm{D}_{\text {mean }}(\%)$ & & & & $67.94 \pm 2.34$ & $84.45 \pm 2.42$ & $81.45 \pm 33.01$ \\
\hline \multirow[t]{6}{*}{ Bolus } & $\mathrm{V}_{95 \%}(\%)$ & $99.55 \pm 0.49$ & $99.84 \pm 0.32$ & $99.93 \pm 0.15$ & & & \\
\hline & $\mathrm{V}_{100 \%}(\%)$ & $90.18 \pm 6.72$ & $89.69 \pm 14.14$ & $97.66 \pm 2.32$ & & & \\
\hline & $\mathrm{V}_{105 \%}(\%)$ & $38.91 \pm 19.06$ & $33.82 \pm 27.57$ & $51.21 \pm 20.24$ & & & \\
\hline & CI & $1.00 \pm 0.00$ & $1.00 \pm 0.00$ & $1.00 \pm 0.00$ & & & \\
\hline & HI & $1.09 \pm 0.01$ & $1.07 \pm 0.01$ & $1.06 \pm 0.02$ & & & \\
\hline & $\mathrm{D}_{\text {mean }}(\%)$ & & & & $98.31 \pm 2.16$ & $101.09 \pm 2.21$ & $102.11 \pm 2.32$ \\
\hline
\end{tabular}

pPTV: Partial planning target volume; pPTV $\mathrm{P}_{0-2 \mathrm{~mm}}$ : volume within $2 \mathrm{~mm}$ of the pPTV; $\mathrm{pPTV}_{2-4 \mathrm{~mm}}$ : volume within 2 to $4 \mathrm{~mm}$ of the pPTV;

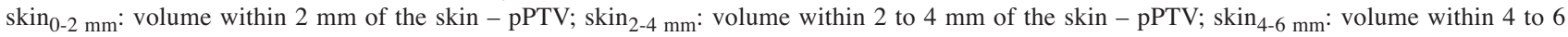
$\mathrm{mm}$ of the skin - pPTV; $\mathrm{V}_{\mathrm{x} \%}$ : volume receiving $\geq \mathrm{x} \%$ of prescribed dose; $\mathrm{D}_{\text {mean }}$ : mean dose for the skin region; CI: conformity index; HI: homogeneity index; sCBS: semi-cylindrical beam spoiler; OB: open beam.

Phantom study. Figure 4 shows the measured and calculated lateral beam profiles in the Rando phantom for a field size of $5 \times 5 \mathrm{~cm}^{2}$ with the $6 \mathrm{MV}$ photon beam. All beam profiles were normalized with respect to the central axis. The lateral dose profiles and 2D dose distributions obtained from the treatment planning system and film measurements using the Rando phantom were in good agreement. The RMSEs between the measured and calculated values for the $\mathrm{OB}, \mathrm{sCBS}$, and bolus were $0.03 \%, 0.04 \%$, and $0.03 \%$, respectively; the maximum differences in the dose values selected within $80 \%$ of the dose at the central axis were $2.2 \%, 1.6 \%$, and $4.6 \%$, respectively. In the build-up regions on both sides, the SCBS showed more effective dose enhancement than the $\mathrm{OB}$ and demonstrated a lower skin dose than that with the bolus. 
Table II. Differences among the three methods with regard to the pDVH parameters.

\begin{tabular}{|c|c|c|c|c|c|c|c|c|}
\hline \multirow[b]{2}{*}{ Plan } & & \multirow[b]{2}{*}{ Parameters } & \multicolumn{6}{|c|}{$p$-Value } \\
\hline & & & pPTV & $\mathrm{pPTV}_{0-2 \mathrm{~mm}}$ & $\mathrm{pPTV}_{2-4 \mathrm{~mm}}$ & 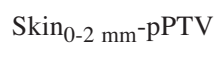 & Skin $_{2-4 ~ m m}$-pPTV & Skin $_{4-6 ~ m m}$-pPTV \\
\hline \multirow[t]{6}{*}{ sCBS $v s . \mathrm{OB}$} & pPTV & V95\% (\%) & $<0.01 *$ & $<0.01^{*}$ & $<0.01 *$ & & & \\
\hline & & $\mathrm{V} 100 \%(\%)$ & $<0.01 *$ & $<0.05^{*}$ & $<0.01 *$ & & & \\
\hline & & $\mathrm{V} 105 \%(\%)$ & 0.261 & 0.786 & 0.464 & & & \\
\hline & & CI & $<0.01 *$ & $<0.01 *$ & $<0.01 *$ & & & \\
\hline & & HI & $<0.01 *$ & $<0.01^{*}$ & $<0.01^{*}$ & & & \\
\hline & skin & $\mathrm{D}_{\text {mean }}(\%)$ & & & & $<0.01 * *$ & $<0.01 * *$ & NS \\
\hline \multirow[t]{6}{*}{ sCBS vs. Bolus } & pPTV & V95\% (\%) & 0.894 & 0.751 & 1.000 & & & \\
\hline & & $\mathrm{V} 100 \%(\%)$ & 0.447 & 0.057 & 0.841 & & & \\
\hline & & V105\% (\%) & 0.247 & 0.246 & 0.119 & & & \\
\hline & & CI & 0.887 & 0.742 & 1.000 & & & \\
\hline & & HI & 0.988 & 0.847 & 1.000 & & & \\
\hline & skin & $\mathrm{D}_{\text {mean }}(\%)$ & & & & $<0.01 * * *$ & $<0.05^{* * *}$ & 0.988 \\
\hline
\end{tabular}

pPTV: Partial planning target volume; $\mathrm{pPTV}_{0-2 \mathrm{~mm}}$ : volume within $2 \mathrm{~mm}$ of the pPTV; pPTV $2-4 \mathrm{~mm}$ : volume within 2 to $4 \mathrm{~mm}$ of the pPTV;

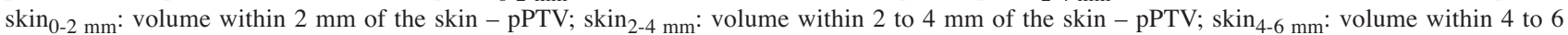
$\mathrm{mm}$ of the skin - pPTV; $\mathrm{V}_{\mathrm{x} \%}$ : volume receiving $\geq \mathrm{x} \%$ of prescribed dose; $\mathrm{D}_{\text {mean }}$ : mean dose for the skin region; CI: conformity index; HI: homogeneity index; sCBS: semi-cylindrical beam spoiler; OB: open beam. *sCBS plan is significantly better than OB plan; **OB plan is significantly better than sCBS plan; ***sCBS plan is significantly better than bolus plan.

Figure 5 shows the results of the gamma index analysis for the measured and calculated $2 \mathrm{D}$ dose distributions with the $6 \mathrm{MV}$ photon beam with and without the sCBS. The average percentage of points passing the gamma criterion was over $98 \%$ for both the sCBS and open field.

Treatment planning study. Table I summarizes the DVH parameters of the pPTVs and Skin-pPTVs for plans conducted using the sCBS, OB, and bolus. For the DVH parameters of the pPTVs, the values of $\mathrm{V}_{95 \%}, \mathrm{~V}_{100 \%}$, and $\mathrm{V}_{105 \%}$ for the sCBS and bolus plans were in good agreement, with no statistically significant differences. However, the values of $\mathrm{V}_{95 \%}$ for the sCBS plans were slightly lower than those for the bolus plans. The differences in the values of the $\mathrm{V}_{95 \%}$ and $\mathrm{V}_{100 \%}$ for the sCBS and OB plans were statistically significant (Table II). The CI and HI of the pPTVs for the sCBS plans were better than those for the OB plans and had statistically significant differences. No differences were observed between the sCBS and bolus plans for the CI and HI. For the DVH parameters of the partial skin regions, the values of the mean dose $\left[D_{\text {mean }}(\%)\right]$ showed statistical significances for the $\mathrm{sCBS} v s$. OB and SCBS vs. bolus plans. In the comparison of the bolus plans, the sCBS plans showed a dose reduction in the $\mathrm{Skin}_{0-2} \mathrm{~mm}^{-} \mathrm{pPTV}$ and $\mathrm{Skin}_{2-4 \mathrm{~mm}^{-}}$ pPTV regions, which exist in the most superficial skin layer.

\section{Discussion}

In this study, a sCBS was designed and fabricated for enhancing the dosage in the build-up region of an applied 6
MV photon beam, while providing better skin sparing effects than a bolus in the treatment of early glottic cancer involving the anterior commissure. In particular, $6 \mathrm{MV}$ or higher-energy $\mathrm{X}$-rays could result in underdosing in the superficial regions owing to inadequate dose build-up at the area of the anterior commissure as well as lack of electronic equilibrium at the air-tissue interface in a field size of $5 \times 5$ or $6 \times 6 \mathrm{~cm}^{2}$, which is commonly used in early glottic cancers treated with megavolt photon beams. For the PDD measurements, sufficient dose at a depth of $0.5 \mathrm{~cm}$ was delivered for greater than $90 \%$ of the spoiler-to-surface distances, including the radius of the inner diameter of the sCBS (Figure 3). For lateral dose distributions calculated and measured using the Rando phantom with the parallel-opposed lateral field, use of the sCBS within the build-up region clearly demonstrated a higher dose than that in the open field without the beam spoiler and lower dose to the skin region than the bolus (Figure 4). Before clinical application of the sCBS, a planning study was conducted to verify its clinical feasibility. In the treatment planning study using the CT data of eight patients, the partialvolume dose histograms showed that the sCBS developed in this study adequately provided the needed dose enhancements within the build-up regions, while preserving the skin sparing effects for typical parallel-opposed lateral fields of early glottic cancer (Table I). In other words, both the sCBS and bolus provide similar dose coverage in the pPTV, but the sCBS has better skin sparing effects.

One option when using the $6 \mathrm{MV}$ beam is to use the bolus to deliver a sufficient dose to the anterior commissure in patients with glottic cancer. This can provide a sufficient 
dose to the tumor area close to the skin, but it is difficult to expect the skin sparing effects. Moreover, since the skin surface is highly curved, it is difficult to set up the bolus to adhere to the skin, and the dose distribution may change owing to the air gap between the bolus and skin. From the results of comparing the doses according to the bolus or beam spoiler, Lee et al. showed that the ideal skin sparing effect could be obtained with a spoiler than the bolus for a similar beam quality (16).

Several researchers have reported the characteristics of using a $10 \mathrm{MV}$ photon beam for patients with glottic cancer $(18,19)$. Kubo et al. reported that even with the $10 \mathrm{MV}$ beam, the beam spoiler can deliver a dose similar to that of Co-60 in the superficial regions (18). In addition, Nroomandrad et al. showed that the use of a spoiler increased the dose in the outfield area (19); this is attributable to the secondary electrons generated in the spoiler that spread out from the field to transmit the required dose. As the spoiler-to-skin distance increases, the secondary electrons tend to spread farther; hence, placing the spoiler close to the skin can minimize unnecessary dose delivery to the outfield areas.

Recently, intensity-modulated radiotherapy (IMRT) and volumetric-modulated arc therapy (VMAT) have been regarded as the main treatment techniques at all sites because IMRT or VMAT has superior dose distribution compared to the three-dimensional conformal radiation therapy (3D CRT) and can reduce unnecessary dose delivery to the surrounding normal tissues, thereby increasing target coverage by injecting beams from various directions. The planar-type beam spoiler is applicable when two opposing bilateral fields are used; however, it has the disadvantage of not being applicable when using beams of various angles, such as those in IMRT and VMAT. In addition, even in the case of the 3D CRT, the cylindrical-type beam spoiler developed in this study is optimal for ensuring that the angle between the beam and spoiler is constant when using a non-bilateral angle to minimize the dose delivered to major organs, such as the carotid artery. The cylindrical beam spoiler also has the advantages of being able to change the skin surface dose via thickness adjustments based on factors, such as the location of the target volume, and easy patient setup. Therefore, in the treatment of early glottic cancers using the $6 \mathrm{MV}$ photon beam, the cylindrical beam spoiler can be an effective tool in the clinical environment.

\section{Conclusion}

In this study, we investigated the beam dosimetry characteristics in the build-up region and clinical feasibility of a sCBS for the treatment of early glottic cancer. The sCBS yields an improved build-up to the existing $6 \mathrm{MV}$ beam, while providing increased skin sparing effects compared to the application of a bolus. The proposed technique offers sufficient dose delivery even for target volumes that are close to the skin, such as the anterior commissure, when employing the parallel-opposed lateral fields of the $6 \mathrm{MV}$ photon beam. This sCBS is also preferable to the existing parallel-opposed planar-type spoilers applied in head and neck cancer treatments and the conventional bolus in terms of ease of application for patient set-up in clinical practice. The sCBS can thus be a useful option for the radiation treatment of early glottic cancers, especially those involving the anterior commissure.

\section{Conflicts of Interest}

The Authors declare no conflicts of interest in relation to this study.

\section{Authors' Contributions}

Conceptualization: SSS and WSA; methodology: WSA and SA; data curation: WSA; forma analysis: SSS; funding acquisition: WSA and SA; investigation: SHB, SSS and WSA; supervision: WSA; validation: JSK and SA; visualization: SHB; writing-original draft: SSS, SA and SHB; wright-review \& editing: WSA, SSS and SA.

\section{Acknowledgements}

This research was supported by the Nuclear Safety Research Program through the Korea Foundation of Nuclear Safety (KoFONS) using financial resources granted by the Nuclear Safety and Security Commission (NSSC) of the Republic of Korea (No. 2003013), National Research Foundation of Korea (NRF) grant funded by the Korean government (MSIT) (No. 2019R1A2C108912912), Basic Science Research Program through the National Research Foundation of Korea (NRF) funded by the Ministry of Education (No. 2018R1D1A1B07050217, No. 2021R1I1A1A01057995) and Medical Research Promotion Program through the Gangneung Asan Hospital funded by the Asan Foundation (No. 2021IC003).

\section{References}

1 Amornmarn R, Prempree T, Viravathana T, Donavanik V and Wizenberg MJ: A therapeutic approach to early vocal cord carcinoma. Acta Radiol Oncol 24(4): 321-325, 1985. PMID: 2994388. DOI: $10.3109 / 02841868509136059$

2 Harwood A, Hawkins N, Rider W and Bryce D: Radiotherapy of early glottic cancer-I. Int J Radiation Oncology Biol Phys 5(4): 473-476, 2018. DOI: 10.1016/0360-3016(79)90808-3

3 Woodhouse RJ, Quivey JM, Fu KK, Sien PS, Dedo HH and Phillips TL: Treatment of carcinoma of the vocal cord. A review of 20 years experience. Laryngoscope 91(7): 1155-1162, 1981. PMID: 7242207. DOI: 10.1288/00005537-198107000-00014

4 Epstein BE, Lee DJ, Kashima H and Johns ME: Stage T1 glottic carcinoma: results of radiation therapy or laser excision. Radiology 175(2): 567-570, 1990. PMID: 2326483. DOI: 10.1148/radiology.175.2.2326483

5 Fein DA, Mendenhall WM, Parsons JT and Million RR: T1-T2 squamous cell carcinoma of the glottic larynx treated with radiotherapy: a multivariate analysis of variables potentially 
influencing local control. Int J Radiat Oncol Biol Phys 25(4): 605-611, 1993. PMID: 8454477. DOI: 10.1016/03603016(93)90005-g

6 Chera BS, Amdur RJ, Morris CG, Kirwan JM and Mendenhall WM: T1N0 to T2N0 squamous cell carcinoma of the glottic larynx treated with definitive radiotherapy. Int J Radiat Oncol Biol Phys 78(2): 461-466, 2010. PMID: 20153124. DOI: 10.1016/j.ijrobp.2009.08.066

7 Cellai E, Chiavacci A and Olmi P: Causes of failure of curative radiation therapy in 205 early glottic cancers. Int J Radiat Oncol Biol Phys 19(5): 1139-1142, 1990. PMID: 2254103. DOI: 10.1016/0360-3016(90)90218-9

8 Johansen LV, Overgaard J, Hjelm-Hansen M and Gadeberg CC: Primary radiotherapy of $\mathrm{T} 1$ squamous cell carcinoma of the larynx: analysis of 478 patients treated from 1963 to 1985 . Int J Radiat Oncol Biol Phys 18(6): 1307-1313, 1990. PMID: 2370181. DOI: 10.1016/0360-3016(90)90302-z

9 Medini E, Medini A, Gapany M and Levitt SH: Radiation therapy in early carcinoma of the glottic larynx T1N0M0. Int J Radiat Oncol Biol Phys 36(5): 1211-1213, 1996. PMID: 8985045. DOI: $10.1016 / \mathrm{s} 0360-3016(96) 00431-2$

10 Nomiya T, Nemoto K, Wada H, Takai Y and Yamada S: Longterm results of radiotherapy for T1a and T1bN0M0 glottic carcinoma. Laryngoscope 118(8): 1417-1421, 2008. PMID: 18528306. DOI: 10.1097/MLG.0b013e3181781791

11 Tong CC, Au KH, Ngan RK, Cheung FY, Chow SM, Fu YT, Au JS and Law SC: Definitive radiotherapy for early stage glottic cancer by $6 \mathrm{MV}$ photons. Head Neck Oncol 4: 23, 2012. PMID: 22607730. DOI: 10.1186/1758-3284-4-23

12 Lim YJ, Wu HG, Kwon TK, Hah JH, Sung MW, Kim KH and Park CI: Long-term outcome of definitive radiotherapy for early glottic cancer: prognostic factors and patterns of local failure. Cancer Res Treat 47(4): 862-870, 2015. PMID: 25687859. DOI: $10.4143 /$ crt.2014.203

13 Tong CC, Au KH, Ngan RK, Chow SM, Cheung FY, Fu YT, Au JS and Law SC: Impact and relationship of anterior commissure and time-dose factor on the local control of T1N0 glottic cancer treated by $6 \mathrm{MV}$ photons. Radiat Oncol 6: 53, 2011. PMID: 21600025. DOI: 10.1186/1748-717X-6-53
14 Devineni V, King K, Perez C, Mittal B, Simpson J and Emami B: Early glottic carcinoma treated with radiotherapy: impact of treatment energy on success rate. Int J Radiation Oncology Biol Phys 24: 185, 2018. DOI: 10.1016/0360-3016(92)90237-C

15 Hsu SH, Roberson PL, Chen Y, Marsh RB, Pierce LJ and Moran JM: Assessment of skin dose for breast chest wall radiotherapy as a function of bolus material. Phys Med Biol 53(10): 25932606, 2008. PMID: 18441412. DOI: 10.1088/0031-9155/53/ $10 / 010$

16 Lee PC, Thomason $\mathrm{C}$ and Glasgow GP: Characteristics of a spoiled 6-MV beam from a dual-energy linear accelerator. Med Phys 20(3): 717-721, 1993. PMID: 8350824. DOI: 10.1118/ 1.597021

17 Kassaee A, Bloch P, Yorke E, Altschuler MD and Rosenthal DI: Beam spoilers versus bolus for $6 \mathrm{MV}$ photon treatment of head and neck cancers. Med Dosim 25(3): 127-131, 2000. PMID: 11025258. DOI: 10.1016/s0958-3947(00)00038-8

18 Kubo H, Russell MD and Wang CC: Use of 10 MV spoiled x ray beam for treatment of head and neck tumors. Int J Radiat Oncol Biol Phys 8(10): 1795-1798, 1982. PMID: 6818189. DOI: 10.1016/0360-3016(82)90304-2

19 Niroomand-Rad A, Javedan K, Rodgers JE and Harter KW: Effects of beam spoiler on radiation dose for head and neck irradiation with 10-MV photon beam. Int J Radiat Oncol Biol Phys 37(4): 935-940, 1997. PMID: 9128972. DOI: 10.1016/ s0360-3016(96)00538-x
Received September 8, 2021

Revised September 30, 2021

Accepted October 1, 2021 